\title{
Loss of $\mathbf{N}$-acetylgalactosaminyltransferase $\mathbf{3}$ in poorly differentiated pancreatic cancer: augmented aggressiveness and aberrant ErbB family glycosylation
}

\author{
Seema Chugh ${ }^{1}$, Jane Meza ${ }^{2}$, Yuri M Sheinin ${ }^{3}$, Moorthy P Ponnusamy ${ }^{1,4,5}$ and Surinder K Batra*,1,4,5 \\ ${ }^{1}$ Department of Biochemistry and Molecular Biology, University of Nebraska Medical Center, Omaha, NE 68198-5870, USA; \\ ${ }^{2}$ Department of Biostatistics, UNMC College of Public Health, UNMC, Omaha, NE 68198-4375, USA; ${ }^{3}$ Department of Pathology \\ and Microbiology, UNMC, Omaha, NE 68198-5900, USA; ${ }^{4}$ Fred and Pamela Buffett Cancer Center, UNMC, Omaha, NE 68198, USA \\ and ${ }^{5}$ Eppley Institute for Research in Cancer and Allied Diseases, UNMC, Omaha, NE 68198-5950, USA
}

Background: Aberrant glycosylation of several proteins underlie pancreatic ductal adenocarcinoma (PDAC) progression and metastasis. O-glycosylation is initiated by a family of enzymes known as polypeptide N-acetylgalactosaminyl transferases (GalNAcTs/GALNTs). In this study, we investigated the role of the O-glycosyltransferase GALNT3 in PDAC.

Methods: Immunohistochemistry staining of GALNT3 was performed on normal, inflammatory and neoplastic pancreatic tissues. Several in vitro functional assays such as proliferation, colony formation, migration and tumour-endothelium adhesion assay were conducted in GALNT3 knockdown PDAC cells to investigate its role in disease aggressiveness. Expression of signalling molecules involved in growth and motility was evaluated using western blotting. Effect of GALNT3 knockdown on glycosylation was examined by lectin pull-down assay.

Results: $\mathrm{N}$-acetylgalactosaminyl transferase 3 expression is significantly decreased in poorly differentiated PDAC cells and tissues as compared with well/moderately differentiated PDAC. Further, knockdown of GALNT3 resulted in increased expression of poorly differentiated PDAC markers, augmented growth, motility and tumour-endothelium adhesion. Pull-down assay revealed that O-glycans ( $\mathrm{Tn}$ and T) on EGFR and Her2 were altered in PDAC cells, which was accompanied by their increased phosphorylation.

Conclusions: Our study indicates that loss of GALNT3 occurs in poorly differentiated PDAC, which is associated with the increased aggressiveness and altered glycosylation of ErbB family proteins.

Pancreatic ductal adenocarcinoma (PDAC) is a highly aggressive gastrointestinal malignancy with a very poor survival rate (i.e., $\sim 8 \%$ 5-year survival rate; Siegel et al, 2016). The lethal nature of this disease is due to its increased metastatic rate, which accounts for the extreme mortality rate among PDAC patients (Nieto et al, 2008; Das and Batra, 2015). To design and create much-needed targeted treatment modalities for PDAC, it is necessary to understand the gene signatures that contribute to its aggressiveness and high metastases. Several studies report the critical involvement of post-translational modifications on proteins during cancer development and progression (Krueger and Srivastava, 2006; Karve and Cheema, 2011). Further, aberrant changes in glycosylation patterns of proteins have been shown to underlie cancer growth and metastases (Hakomori, 2002;

*Correspondence: Professor SK Batra; E-mail: sbatra@unmc.edu

Received 20 March 2016; revised 4 April 2016; accepted 6 April 2016; published online 17 May 2016

(c) 2016 Cancer Research UK. All rights reserved 0007-0920/16 
Chugh et al, 2015). Proteins can undergo $O$ - and $N$-glycosylation, which is determined by the amino acid getting modified by the glycan residues (Stanley, 2011). Mucin-type O-glycosylation is the most common $O$-glycosylation that is initiated in the Golgi apparatus through a large family of 20 enzymes, known as UDP-GalNAc:Polypeptide $N$-acetylgalactosaminyltransferases (i.e., GalNAc-Ts/GALNTs; Bennett et al, 2012). GalNAcT enzymes catalyse the first step in mucin-type $O$-glycosylation, wherein these enzymes add an $\mathrm{N}$-acetylgalactosamine sugar residue to the serine/threonine (Ser/Thr) residues on their specific substrates. This addition results in the formation of a Tn carbohydrate antigen (Ju et al, 2011), which can be either sialylated to form sialyl-Tn (STn) antigen or it can be extended to several core carbohydrate structures (Tran and Ten Hagen, 2013).

Despite of the functional homology, distinct substrate specificity has been reported for GalNAcTs (Raman et al, 2008). For instance, during breast cancer, GalNAc-T6 has been shown to have a critical role in glycosylation of mucin 1 (Park et al, 2010). Another study on hepatocellular carcinoma showed the significant involvement of GalNAc-T2 in glycosylation of the growth factor receptor EGFR (Wu et al, 2011). GalNAc-T2 has also been shown to regulate EGFR glycosylation and activity in oral squamous cell carcinoma. (Lin et al, 2014). GalNAc-T3/GALNT3 enzyme is one of the members of the $O$-glycosylation-initiating GalNAc-T family, which has been shown to be highly expressed in oral squamous cell carcinomas and in ovarian cancer (Wang et al, 2014; Harada et al, 2015). Conversely, in lung adenocarcinoma and colorectal carcinoma, decreased expression of GALNT3 is reported, which is associated with a poor prognosis (Dosaka-Akita et al, 2002; Shibao et al, 2002; Gu et al, 2004). Few studies in pancreatic cancer have shown loss of expression of GALNT3 in poorly differentiated pancreatic cancer (Yamamoto et al, 2004; Li et al, 2011). However, the role of GALNT3 in pancreatic cancer progression and metastasis has not been explored much.

The present study elucidates a novel role the loss of GALNT3 has in PDAC aggressiveness. The results from the present study evidence the association between the loss of $O$-glycosyltransferase GALNT3 and an altered glycosylation of ErbB receptors, accompanied by the increased phosphorylation of these growth factor receptors, leading to the increased PDAC aggressiveness. Altogether, our study for the first time showed the pathobiological implications of loss of GALNT3 in poorly differentiated PDAC.

\section{MATERIALS AND METHODS}

Tissue samples from PDAC patients. After approval of institutional review board, formalin-fixed paraffin-embedded tissue specimens from pancreatic cancer patients who underwent Whipple surgery were retrieved. In addition, we also obtained tissue arrays (US Biomax-PA 1002a A009 and BIC14011) that were representative of normal pancreatic tissues, chronic pancreatitis, pancreatic intraepithelial precursor lesions (PanINs) and PDAC (i.e., well-differentiated, moderately differentiated and poorly differentiated cancer).

Cell lines and cell culture. Human microvascular endothelial cells (HMEC-1) were a kind gift from Dr Rakesh Singh (University of Nebraska Medical Center) and were maintained in 5\% RPMI supplemented with L-glutamine. Human PDAC cell lines CD18/ HPAF, BxPC3, Capan-1, and T3M4 were obtained from the American Type Culture Collection (ATCC, Manassas, VA, USA) and grown in recommended media supplemented with antibiotics (10\% DMEM for CD18/HPAF, Capan-1 and T3M4 cells, and 10\% RPMI for BxPC3 cells). These PDAC cells were maintained in a humidified, $5 \% \mathrm{CO}_{2}$ atmosphere at $37^{\circ} \mathrm{C}$. All of these cell lines were tested mycoplasma free before conducting the experiments.
Reagents and antibodies. Supplementary Tables 1 and 2 outline a thorough list of other antibodies and primers used in the study. Biotinylated VVA (binds Tn carbohydrate antigen) and PNA (binds $\mathrm{T}$ carbohydrate antigen) lectins were purchased from Vector Laboratories (Burlingame, CA, USA).

Immunohistochemistry. Immunohistochemistry (IHC) staining was performed on Whipple samples and tissue arrays to analyse GALNT3 expression using a previously described method (Kaur et al, 2014). In brief, after deparaffinisation and dehydration of tissue sections, endogenous peroxidase activity in these human tissues was blocked by $3 \% \mathrm{H}_{2} \mathrm{O}_{2}$ for $1 \mathrm{~h}$. Further, antigen retrieval is done by incubating these tissues with $0.01 \mathrm{M}$ citrate buffer ( $\mathrm{pH}$ 6.8). Subsequently, the tissue slides were blocked with horse serum, which was followed by their incubation with anti-GALNT3 antibody for $16 \mathrm{~h}$ in a humidified chamber at $4{ }^{\circ} \mathrm{C}$. The specificity of the antibody was confirmed using isotype control (Supplementary Figure 1b). The sections were probed with Streptavidin HRP and counterstained with haematoxylin. Expression of GALNT3 was analysed by a pathologist. An intensity (0-no staining, 1-weak staining, 2-moderate staining and 3-strong staining) and percentage of positive cells (range 1-4: $0-25 \%$ positive cells, equal score of $1 ; 26-50 \%$, equal score of 2 ; $51-75 \%$, equal score of 3 ; and $76-100 \%$, equal score of 4 ) were recorded. A composite score obtained by multiplying the two values was assigned, and ranged from 0 to 12 .

Stable GALNT3 knockdown PDAC cells. Stable transfection of GALNT3 was performed by inserting a specific shRNA sequence $\left(5^{\prime}\right.$-GGTCTGATCACTGCTCGGT-3') in the following four PDAC cell types: CD18/HPAF, BxPC3, Capan-1 and T3M4 cells. The Phoenix Packaging cell line was transfected with scramble control (Scr) and pSUPER-Retro-sh-GALNT3 using Lipofectamine 2000 (Invitrogen, Carlsbad, CA, USA). Viral particles collected 48-h post transfection were used to infect CD18/HPAF, BxPC3, Capan-1 and T3M4 cells. Stably transfected, pooled populations of GALNT3 knockdown cells were obtained using the antibiotic selection (Puromycin $4 \mu \mathrm{g} \mathrm{ml}^{-1}$ ). $N$-acetylgalactosaminyl transferase 3 knockdown cells were maintained in $1 \mu \mathrm{g} \mathrm{ml}^{-1}$ puromycinselection media.

Immunofluorescence. Confocal analysis of GALNT3 expression was carried out in CD18/HPAF, T3M4 and Panc-1 PDAC cells. All of these PDAC cells were grown in a 12 -well plate dish to $60 \%$ confluence on autoclaved cover slips for $48 \mathrm{~h}$. After their fixation with $4 \%$ paraformaldehyde $(10 \mathrm{~min})$, these PDAC cells were permeabilised with $0.2 \%$ Triton-X (15 min). Blocking was completed using $10 \%$ bovine serum albumin (BSA; Jackson Immunoresearch Labs, Inc., West Grove, PA, USA), which was followed by overnight incubation with a GALNT3 antibody at $4{ }^{\circ} \mathrm{C}$. The cells were then washed with phosphate-buffered saline (PBS) for $3 \times 5 \mathrm{~min}$ and incubated in dark for $30 \mathrm{~min}$ with FITCconjugated anti-mouse secondary antibody (Jackson Immunoresearch Labs, Inc.) at room temperature. Cells were washed again $(5 \times 5 \mathrm{~min})$ in PBS and mounted on glass slides in vectashieldmounting medium that contained the nuclear staining dye DAPI (Vector Laboratories).

Real-time PCR. RNA is collected from GALNT3 knockdown cells using RNAeasy kit (Qiagen, Valenica, CA, USA). Specifically, $1 \mu \mathrm{g}$ of the RNA was converted to CDNA, which was further used for RT-PCR quantification of GALNTs and epithelial-mesenchymal transition (EMT)-related genes.

Tumour-endothelium cell adhesion assay. Adhesion of cancer cells to activated endothelial cells was examined using CytoSelect Tumour-Endothelium adhesion assay kit (Cell Biolabs, San Diego, CA, USA) (Gu et al, 2010). In brief, 10000-20000 HMEC-1 endothelial cells were cultured on collagen/gelatin-coated 96-well 
plates (Black/Clear Flat Bottom-Corning, Corning, NY, USA) till the formation of monolayer. $\mathrm{N}$-acetylgalactosaminyl transferase 3 scrambled and knockdown cells (CD18/HPAF and BxPC3) suspension pre-labelled with CytoTracker was placed in 96-well plates containing endothelial cells, and incubated at $37^{\circ} \mathrm{C}$ for $1 \mathrm{~h}$. Ninetysix-well plates were then washed three times to remove non-adherent cells. Cell adhesion of labelled scrambled control and GALNT3 knockdown cells was determined by measuring the fluorescence using the fluorescent plate reader at an excitation wavelength of $480 \mathrm{~nm}$ and an emission of $520 \mathrm{~nm}$. Percentage adhesion is calculated as follows: \% cell adhesion $=$ mean fluorescence intensity of experimental wells/mean fluorescence intensity of total cells plated $\times 100 \%$

Lectin pull-down assay and immunoprecipitation. Protein lysates $(600 \mu \mathrm{g})$ collected from scramble control and GALNT3 knockdown cells were incubated with biotinylated VVA $(5 \mu \mathrm{g})$ and PNA $(5 \mu \mathrm{g})$ lectins to detect $\mathrm{Tn}$ and $\mathrm{T}$ carbohydrate antigens, respectively, located on glycoproteins. Proteins bound to these biotinylated lectins were then pulled down using streptavidin agarose $(20 \mu \mathrm{l})$ as described previously (Seales et al, 2003). Pulled down proteins were then immunoblotted with EGFR and Her2.

Colony formation assay. Colony formation assays were performed in scramble control and GALNT3 knockdown cells using standard protocols (Bafna et al, 2008). In brief, 500-1000 cells were seeded in six-well plates and allowed to adhere overnight, following which media was changed to $1 \%$, and the colony formation efficiencies of the cells were analysed after 3-4 weeks by staining them with crystal violet $(0.1 \%$, w/v) in $20 \mathrm{nM} 4$-morpholinepropanesulfonic acid (Sigma Chemicals, St Louis, MO, USA).

WST-1 proliferation assay. Effect of GALNT3 knockdown on cell growth was assessed using WST-1 assay. For this assay, 1000 cells were plated onto 96-well plates and allowed to adhere overnight. On the next day, the media was changed to $1 \%$ DMEM, and WST1 reagent was added to each well. Absorbance was measured at $450 / 630 \mathrm{~nm} 3 \mathrm{~h}$ after WST-1 addition. Similarly, readings taken for another 3 consecutive days (Torres et al, 2013).

Wound-healing assay. Cell motility was evaluated in GALNT3 knockdown cells using a wound-healing assay as described previously (Seshacharyulu et al, 2015). Wound closure was evaluated in scramble control and GALNT3 knockdown cells using Image J software (https://imagej.nih.gov/ij/).

Western and lectin blots. Western blotting was performed using standard procedures and manufacturer's protocols. In brief, lysates were collected from PDAC cells (CD18/HPAF, BxPC3, Capan-1, Panc-1, T3M4, Aspc-1, Colo-357 and MiaPaCa) and GALNT3 knockdown PDAC cells (BxPC3, CD18/HPAF, T3M4 and Capan-1) $48 \mathrm{~h}$ after plating. The above-listed lysates $(40 \mu \mathrm{g})$ were probed for GALNT3 expression using 10\% SDS/PAGE. Furthermore, GALNT3 knockdown cells were probed for several other oncogenic signalling proteins using antibodies listed in Supplementary Table 1. After appropriate incubation of the secondary antibody, bands were visualised using enhanced chemiluminescence (ECL; Thermo Scientific, Waltham, MA, USA). For lectin blotting, 2\% BSA (Jackson Immunoresearch Labs, Inc.) was used for blocking, and streptavidin HRP was applied to visualise bands using an ECL method (Gnanapragassam et al, 2013).

Statistical analysis. For IHC, Student's $t$-test was used to determine the statistical significance of variation in GALNT3 composite score among different disease groups. Similarly, for all the functional assays, Student's $t$-test was used to determine statistical significance. A $P$-value of $<0.05$ was considered statistically significant.

\section{RESULTS}

Differential expression of GALNT3 in PDAC cells. To investigate the expression of GALNT3 during PDAC, a panel of PDAC cell lines derived from well-, moderate- and poorly differentiated carcinomas was used. $\mathrm{N}$-acetylgalactosaminyl transferase 3 protein expression was increased in well- and moderately differentiated PDAC cells (CD18/HPAF, Capan-1, SW1990, Colo-357 and BxPC3) as shown by western blot analysis, whereas poorly differentiated PDAC cells displayed no expression of GALNT3 (AsPC-1, Panc-1 and MIA PaCa-2; Figure 1A). To confirm these findings, immunofluorescence staining of GALNT3 expression was performed for certain PDAC cells with varied differentiation status (Supplementary Figure 1a). In accordance with the western blot data, immunofluorescence studies also demonstrated loss of GALNT3 in poorly differentiated PDAC cells. These results suggest that GALNT3 is differentially expressed in PDAC cells.

Increased GALNT3 expression during PDAC progression from PanINs to well/moderately differentiated PDAC, but subsequent loss in poorly differentiated PDAC tissues. To further evaluate the relationship between GALNT3 expression and disease progression, IHC staining of GALNT3 was conducted in commercially available tissue arrays comprised of normal pancreatic tissues, samples procured from patients with chronic pancreatitis, PanINs and PDAC (well differentiated, moderately differentiated and poorly differentiated). Immunohistochemistry staining was also performed on archived PDAC tissues procured via the Whipple procedure. Granular cytoplasmic pattern of staining was observed in tissues positive for GALNT3 expression. Normal pancreatic ducts were negative for GALNT3 expression (weak staining in islets of Langerhans; Figure 1C). Majority of chronic pancreatitis and PanINs samples showed negative staining, though weak staining was observed in few PanINs ducts (Supplementary Table 3). Compared with PanIN lesions and chronic pancreatitis, the composite score for GALNT3 increased significantly $(P<0.001)$ in the well- and moderately differentiated PDAC samples. However, the composite score decreased significantly $(P<0.05)$ in poorly differentiated cancer (Figure 1B). Overall, these results demonstrate that loss of GALNT3 expression occurs primarily during advanced and poorly differentiated PDAC, and not in moderately or well-differentiated PDAC (representative picture-Figure 1C; Supplementary Figure 1c).

GALNT3 knockdown and decreased expression of Tn carbohydrate antigen. To gain insight into the role of GALNT3 in PDAC progression, knockdown of GALNT3 was performed using specific shRNA in the following four different PDAC cell lines: CD18/HPAF, BxPC3, Capan-1 and T3M4. Knockdown was confirmed by western blot analysis and real-time PCR (Figure 2A; Supplementary Figure 1d). As GALNT3 is the first enzyme in the $O$-glycosylation pathway and catalyses the formation of $\mathrm{Tn}$ carbohydrate antigen, we next evaluated the expression of the Tn carbohydrate antigen in GALNT3 knockdown PDAC cells. VVA lectin blot demonstrated a slight decrease in expression of Tn carbohydrate antigen in GALNT3 knockdown PDAC cells (Figure 2B).

Increased expression of poorly differentiated PDAC cell markers with loss of GALNT3. Considering that loss of GALNT3 expression occurred in poorly differentiated PDAC cells and tissues, the expression of poorly differentiated PDAC cell markers was examined in GALNT3 knockdown PDAC cells. However, no defined markers exist to characterise well-, moderateand poorly differentiated forms of PDAC. On the other hand, previous studies show that expression of the protein Sox 2 increases in poorly differentiated PDAC cells (Ben-Porath et al, 2008; 

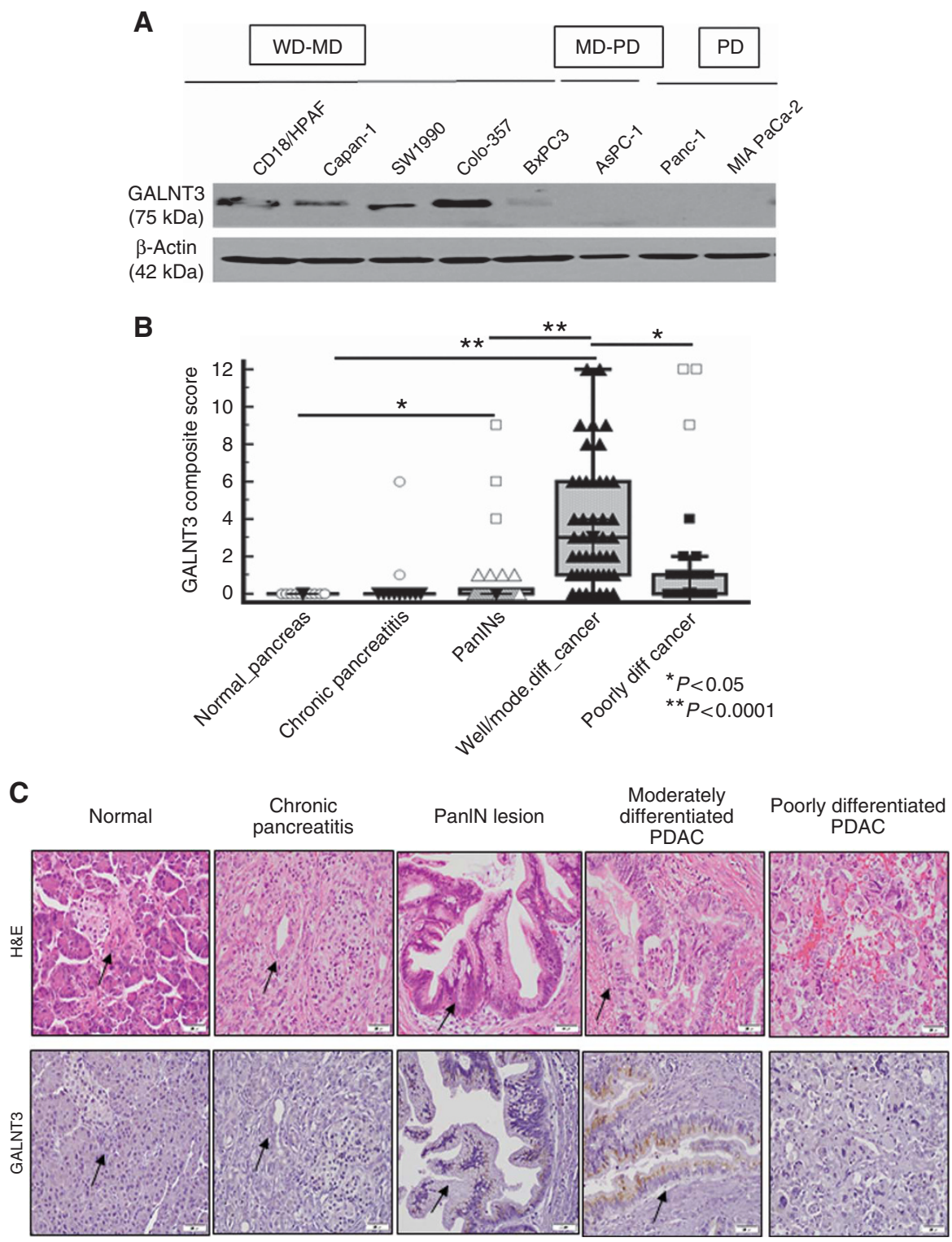

Figure 1. Differential expression of GALNT3 in PDAC cells and tissues. (A) Western blot analysis of GALNT3 expression in a panel of eight PDAC cell lines demonstrates loss of GALNT3 expression in poorly differentiated PDAC cells WD, well differentiated; MD, moderately differentiated; PD, poorly differentiated). (B) Immunohistochemistry (IHC) staining performed on tissues representing chronic pancreatitis, PanIN lesions and different grades of PDAC revealed progressive increase of composite score of GALNT3 from chronic pancreatitis and PanIN lesions to well-differentiated PDAC; however, the composite score is decreased in poorly differentiated PDAC. (C) Representative images of GALNT3 immunohistochemical staining in pancreatic cancer progression tissues-upper panel represents H\&E staining of normal, chronic pancreatitis, PanIN, moderately and poorly differentiated pancreatic cancer tissues, whereas lower panel represents the corresponding GALNT3 staining in these tissues (figure magnification$\times 400$; scale bar, $50 \mu \mathrm{m}$; black arrows indicate ductal regions).

Herreros-Villanueva et al, 2013). Another study showed loss of the carbohydrate antigen sialyl Lewis a (SLe $\mathrm{a} / \mathrm{CA}-19.9)$ occurred in poorly differentiated PDAC (Steinberg, 1990). In turn, the expression of Sox 2 and SLe ${ }^{a}$ was evaluated in GALNT3 knockdown PDAC cells. Data showed increased expression of Sox 2 and decreased expression of SLe ${ }^{\mathrm{a}}$ in GALNT3 knockdown PDAC cells (Figure 2C and D).

Increased PDAC cell growth and proliferation with GALNT3 knockdown. In our results, loss of GALNT3 is observed in the poorly differentiated PDAC and therefore the effect of GALNT3 knockdown on disease aggressiveness was further examined in the study herein. Specifically, in vitro assays were used to examine the effect of GALNT3 knockdown on PDAC cell growth and proliferation. WST-1 assay results demonstrated significant increase in proliferation of GALNT3 knockdown CD18/HPAF cells (Figure 3A). GALNT3 knockdown also increased proliferation of Capan-1 PDAC cells ( $P$-value $<0.05$; Supplementary Figure 2a). Further, increased numbers of colonies were observed in GALNT3 knockdown cells compared with scramble control cells ( $P$-value $<0.05$; Figure 3B and C; Supplementary Figure 2b). In addition, increased proliferation and numbers of colonies in GALNT3 knockdown PC cells were also associated with increased expression of Cyclin-A (Figure 3D). Overall, these findings suggest that loss of GALNT3 expression leads to increased proliferation and growth of PDAC cells.

Increased motility and endothelial cell adhesion of GALNT3 knockdown PDAC cells. To determine the effect that GALNT3 has on cancer cell mobility, wound-healing assays were conducted 
A

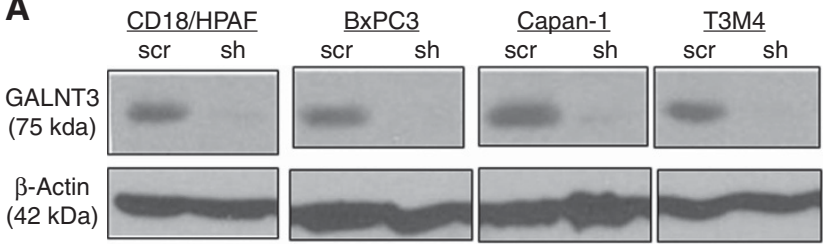

B
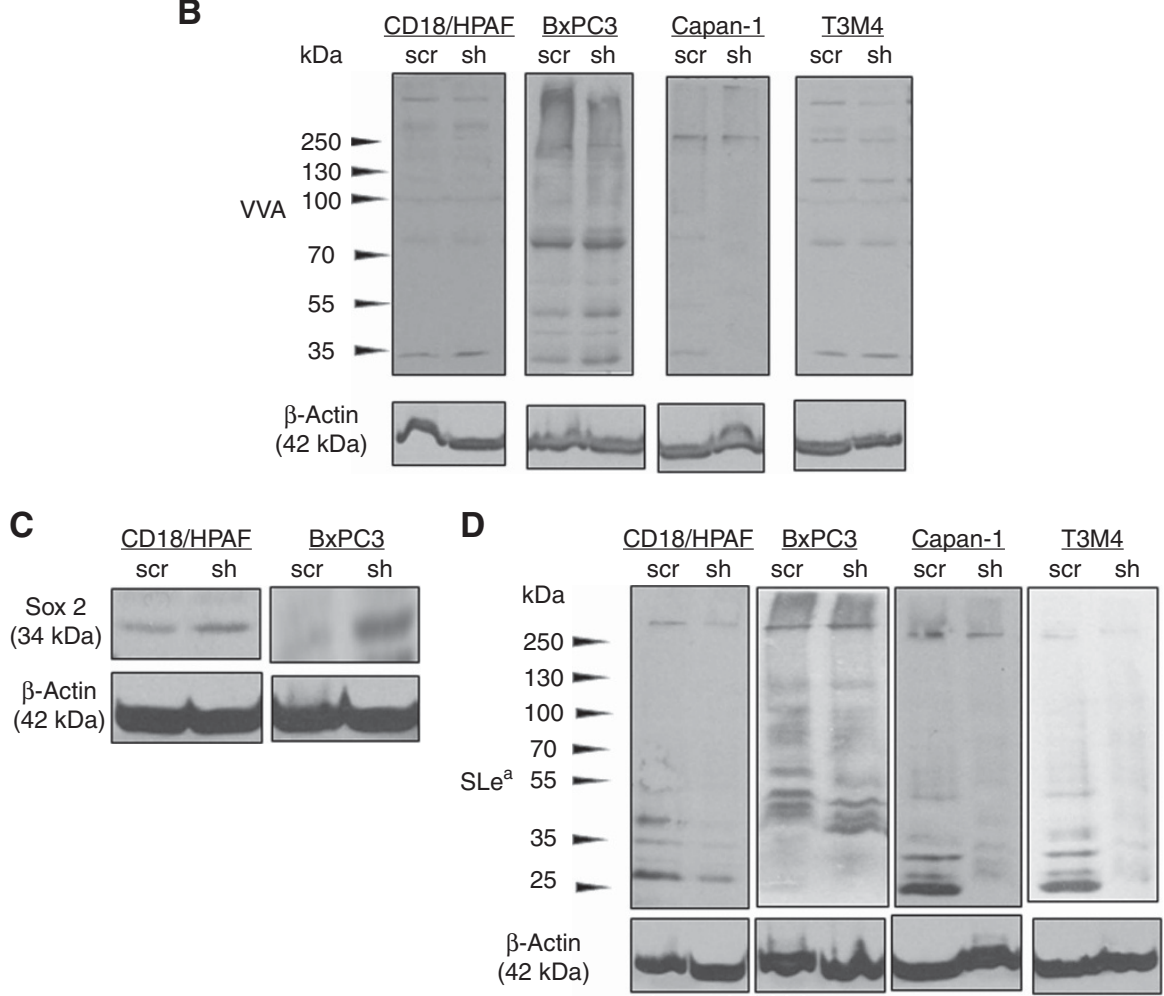

Figure 2. Altered expression of poorly differentiated PDAC cell markers in GALNT3 knockdown cells. (A) Stable knockdown of GALNT3 was carried out using GALNT3-specific shRNA in four different PDAC cells_CD18/HPAF, BxPC3, Capan-1 and T3M4. Beta-actin was used as a loading control. (B) WA lectin blot demonstrates decrease of Tn carbohydrate antigen in GALNT3 knockdown PDAC cells. (C and D) Immunoblot analysis shows altered expression of differentiation markers with increased expression of Sox2 and decreased expression of SLe ${ }^{a}$ in GALNT3 knockdown PDAC cells.

for GALNT3 knockdown CD18/HPAF and BxPC3 PDAC cells. Notably, compared with scramble control cells, GALNT3 knockdown significantly increased the percentage of wound closure after $24 \mathrm{~h}(P$-value $<0.05$; Figure $4 \mathrm{~A})$. To further assess the effect that GALNT3 has on cancer cell mobility, the expressions of EMT proteins involved in increased cell motility (e.g., E-cadherin, $\mathrm{N}$-cadherin, Vimentin and ZEB-1) were examined. Interestingly, increased expression was seen for mesenchymal markers $(N$-cadherin, vimentin and Zeb-1), and decreased expression was seen for epithelial marker (E-cadherin) in GALNT3 knockdown PDAC cells (Figure 4B; Supplementary Figure $2 \mathrm{c}$ and e). Further, GALNT3 knockdown CD18/HPAF and BxPC3 PDAC cells also showed EMT-like cell morphology (Figure 5A).

Next, we evaluated the expression of the terminal carbohydrate epitope, sialylated Lewis $\mathrm{x}, \mathrm{SLe}^{\mathrm{x}}$, for which a study by Kawarada et al (2000) demonstrated its involvement in metastasis. Data from our study revealed increased expression of SLe ${ }^{\mathrm{x}}$ in GALNT3 knockdown CD18/HPAF and BxPC3 PDAC cells (Figure 5B). In addition, GALNT3 knockdown CD18/HPAF and Capan-1 PDAC cells showed increased expression of sialylated Tn carbohydrate antigen (STn; Supplementary Figure 2d). Likewise, prior studies indicate that the negative charge imparted by sialic acid residues allows cancer cells to detach from each other, thus aiding in metastatic dissemination (Schultz et al, 2012; Hauselmann and
Borsig, 2014). Increases in SLe ${ }^{\mathrm{x}}$ and STn carbohydrate antigens in GALNT3 knockdown PDAC cells suggest that there is not a complete abrogation of glycosylation in GALNT3 knockdown cells, which is potentially explained by the compensatory action of other GALNTs. For example, increased expressions of few GALNT genes were seen in GALNT3 knockdown cells (such as GALNT2, GALNT10 and GALNT11, GALNT12, GALNT13 and GALNT14; Supplementary Figure $3 \mathrm{a}$ and $\mathrm{b}$ ). SLe ${ }^{\mathrm{x}}$ carbohydrate antigen has been shown to facilitate cancer cells to cross the endothelium barrier through its interaction with E-selectin on endothelial cells, therefore to investigate the functional impact of increased SLe ${ }^{\mathrm{x}}$ expression, we examined the adhesion of GALNT3 knockdown PDAC cells to endothelium cells (Takahashi et al, 2001; St Hill, 2011). Interestingly, GALNT3 knockdown CD18/HPAF and BxPC3 cells showed significantly increased adhesion to endothelium cells as compared with scrambled control cells (Figure 5C). These results suggest that GALNT3 knockdown cells might have increased propensity to metastasise.

GALNT3 knockdown and altered expression of several oncogenic signalling proteins. To gain insight into the mechanisms involved in the increased aggressiveness of PDAC, we investigated the expression of ErbB proteins associated with increased tumour malignancy. Increased phosphorylation of ErbB 


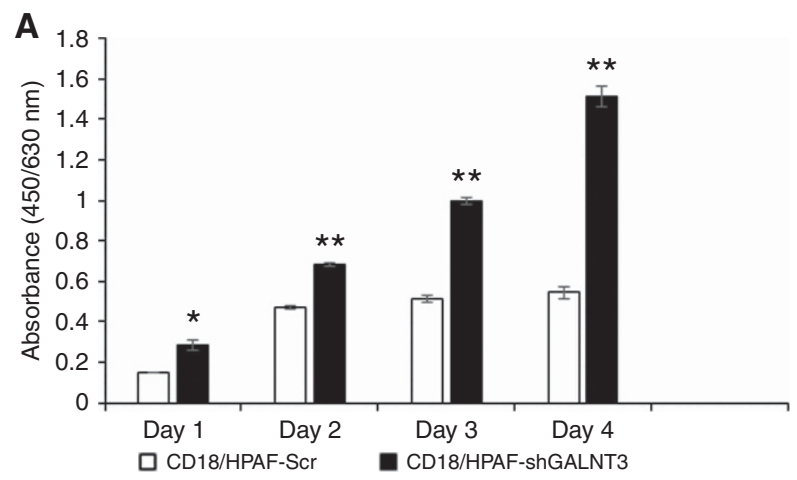

B

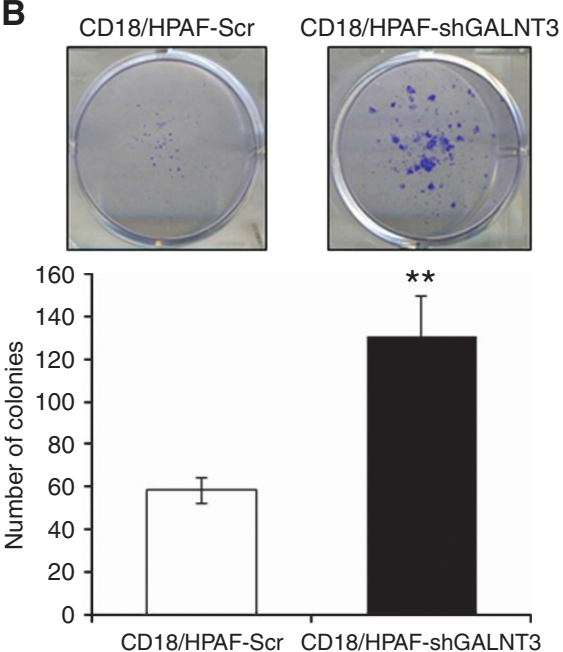

C

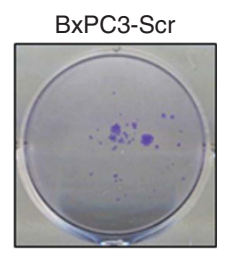

BxPC3-shGALNT3
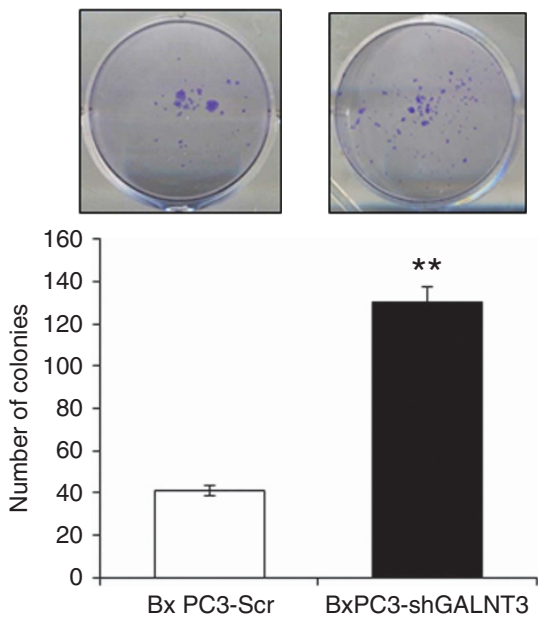

Bx PC3-Scr BxPC3-shGALNT3

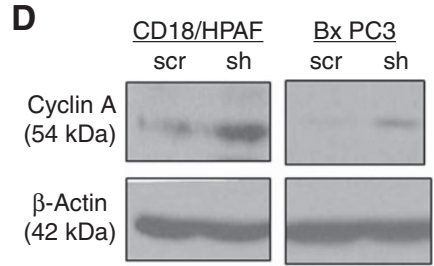

Figure 3. Effect of GALNT3 knockdown on growth and proliferation of PDAC cells. (A) Bar graph representing significant increase in proliferation of GALNT3 knockdown CD18/HPAF cells ( ${ }^{*}<<0.001$ ) as demonstrated by WST-1 cell proliferation reagent. (B, C) Representative pictures of colony formation assay in CD18/HPAF and BxPC3 cells show significant increase in number of colonies (**P<0.001) in GALNT3 knockdown PDAC cells. (D) Increased expression of cell cycle regulatory protein, Cyclin-A in GALNT3 knockdown cells.

family of membrane receptors has been implicated in PDAC growth and motility (Lakshmanan et al, 2015; Seshacharyulu et al, 2015). Data from the present study showed increased phosphorylation of ErbB family members, including EGFR, Her2 and Her3, in GALNT3 knockdown PDAC cells compared with scrambled control cells (Figure 6A).

GALNT3 knockdown and altered glycosylation of EGFR and Her2. To delineate the specific mechanisms by which increased phosphorylation of ErbB family members occurs; the effect of GALNT3 knockdown on O-glycosylation modifications on these proteins was investigated. Previous studies evidence the involvement of altered $O$-glycosylation in regulating activation of EGFR (Wu et al, 2011; Lin et al, 2014; Huang et al, 2015). Therefore, to examine the effect of GALNT3 knockdown on Tn and T carbohydrate antigens associated with EGFR and Her2, cell lysates from scramble control and GALNT3 knockdown cells were pulled down using biotinylated VVA (binds Tn carbohydrate antigen) and PNA lectins (binds T carbohydrate antigen; Figure 6B). VVA and PNA pull-down lysates were further probed with EGFR and
Her2/Neu to examine Tn and $\mathrm{T}$ carbohydrate alterations on these receptors. Lectin pull-down assay demonstrated increased Tn and $\mathrm{T}$ carbohydrate antigens on EGFR and Her2 in GALNT3 knockdown CD18/HPAF and BxPC3 cells (Figure 6B). Increased $\mathrm{Tn}$ and $\mathrm{T}$ carbohydrate antigens on these growth receptors in GALNT3 knockdown cells suggest the possible involvement of other GALNTs in EGFR glycosylation in aggressive PDAC. Interestingly, we observed upregulation of GALNT2, which has been shown to enhance the invasive potential of oral squamous cell carcinoma by regulating EGFR glycosylation (Supplementary Figure 3a and b) (Lin et al, 2014). However, the role of GALNT2 in EGFR glycosylation in PDAC needs to be explored.

\section{DISCUSSION}

Deregulated $O$-glycosylation of several proteins underlies cancer progression and metastasis. Furthermore, altered expression of glycosyltransferases leads to aberrant glycosylation of proteins, thus affecting their function. However, specific glycosyltransferases 
A
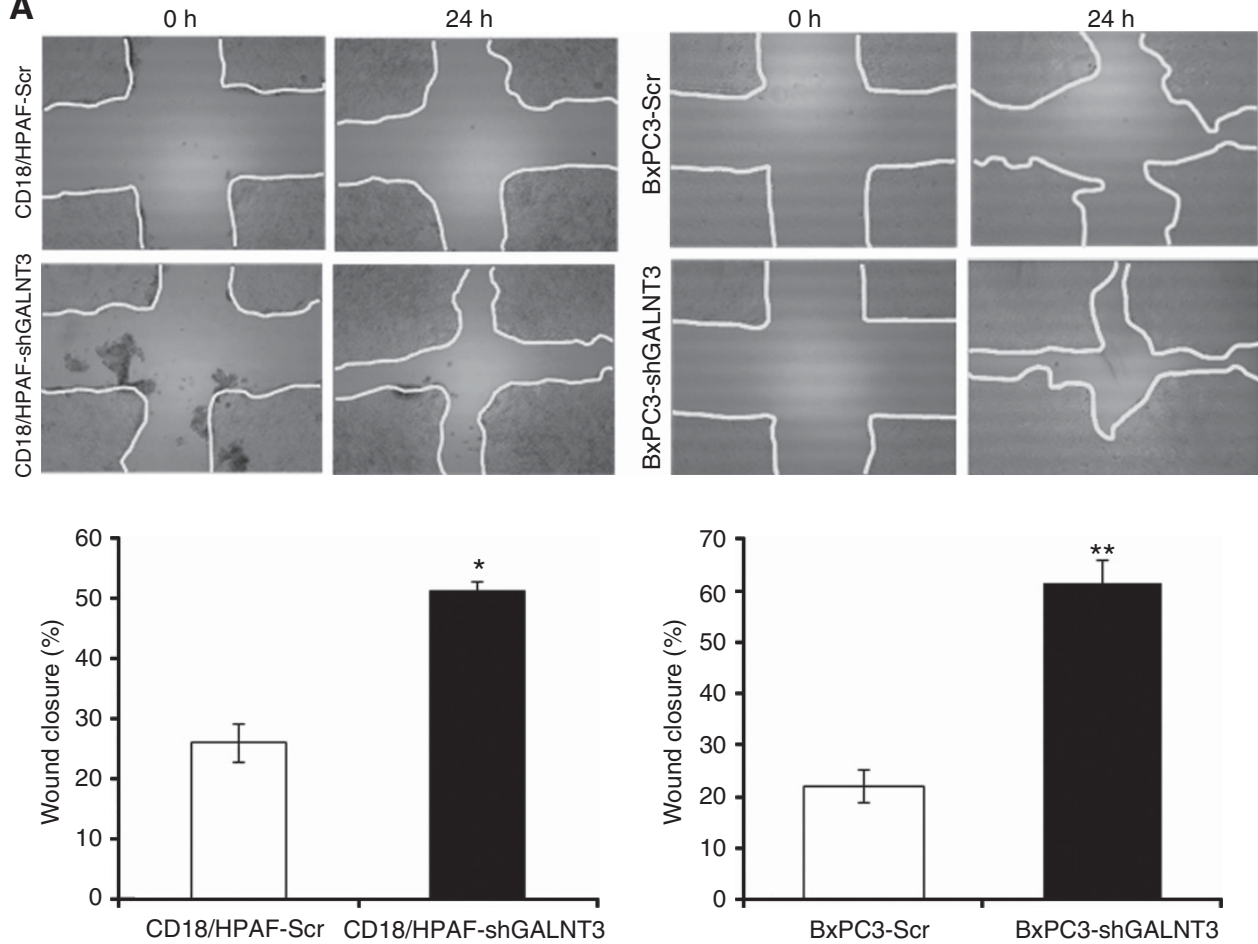

B

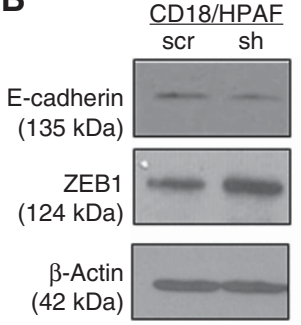

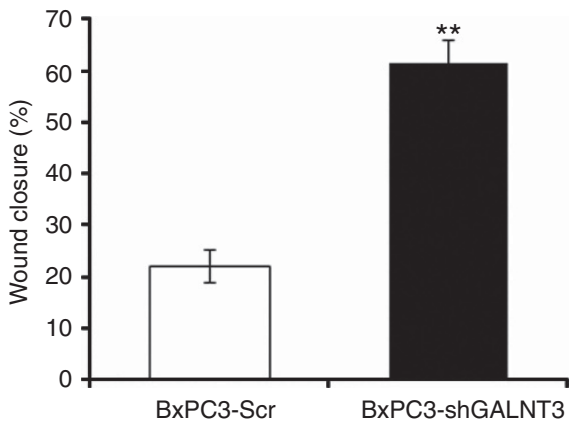

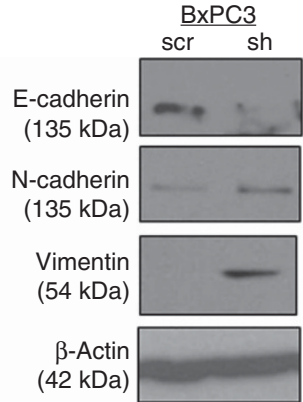

Figure 4. Augmented motility of GALNT3 knockdown PDAC cells. (A) Increased percentage of wound closure of GALNT3 knockdown cells after $24 \mathrm{~h}$. Marked areas show places of wound closure in scramble control and GALNT3 knockdown cells. Bar graphs represent a significant increase in the percentage of wound closure in GALNT3 knockdown CD18/HPAF and BxPC3 cells ( ${ }^{\star} P<0.05$ for CD18/HPAF, ${ }^{\star \star} P<0.001$ for BxPC3 PDAC cells). (B) Altered expression of EMT markers in GALNT3 knockdown PC cells.

related to the increased tumour aggressiveness have been unidentified. We identified that the O-glycosylation-initiating enzyme, GALNT3 is differentially expressed in PDAC. Using IHC staining, we found that GALNT3 expression decreased markedly in poorly differentiated PDAC. Furthermore, we have analysed some of the poorly differentiation markers in GALNT3 knockdown PDAC cells to prove the concept. However, there are no well-defined markers that can filter poorly differentiated PDAC from moderately or well-differentiated PDAC. On the other hand, recent studies on invasive and poorly differentiated PDAC showed increased expression of the transcription factor SOX2 (Ben-Porath et al, 2008; Herreros-Villanueva et al, 2013). In turn, we investigated whether loss of GALNT3 leads to increased expression of SOX2, thus serving as a marker for poorly differentiated PDAC cells. Interestingly, GALNT3 knockdown cells showed increased expression of SOX2. Further, we examined the expression of the carbohydrate antigen SLe ${ }^{\mathrm{a}}$ in GALNT3 knockdown PDAC cells. SLe $^{\mathrm{a}}$, also known as CA-19.9, is a clinically important prognostic marker for PDAC. Still, poorly differentiated PDAC cells have been shown to produce reduced amounts of CA-19.9 as compared with well- or moderately differentiated PDAC cells (Steinberg, 1990). Expression of the carbohydrate antigen SLe ${ }^{\mathrm{a}}$ was found to be decreased in GALNT3 knockdown PDAC cells as compared with scramble control cells. These results suggest that knockdown of GALNT3 in PDAC cells might lead to the poorly differentiated state. Our findings are consistent with previous studies also reporting loss of GALNT3 in other poorly differentiated cancers, including thyroid, gastric and colorectal carcinoma (Shibao et al, 2002; Onitsuka et al, 2003; Mochizuki et al, 2013). For instance, GALNT3 expression in thyroid carcinoma is indicative of its differentiation status. Notably, poorly differentiated components of papillary thyroid carcinoma were found to be less positive for GALNT3 expression compared with well-differentiated components (Mochizuki et al, 2013). On the basis of these supporting studies, our results strongly suggest that GALNT3 is decreased in poorly differentiated PDAC.

In 2011, a study by Li et al investigated the clinical significance of GALNT3 and GALNT6 in PDAC. Their results demonstrated negative staining for GALNT3 expression in poorly differentiated PDAC; however, expression pattern of GALNT3 in different stages of disease progression such as PanINs and chronic pancreatitis was not examined (Li et al, 2011). Further, the functional role of loss of GALNT3 expression in poorly differentiated pancreatic cancer has not been described in this study. Hence, the objective of our study 
A

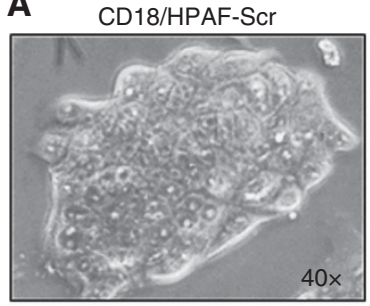

BXPC3-GALNT3-Scr

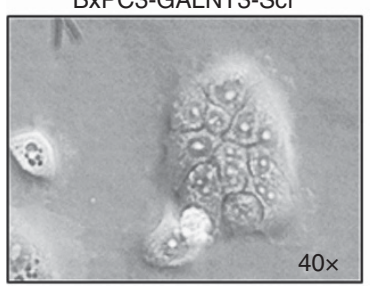

C

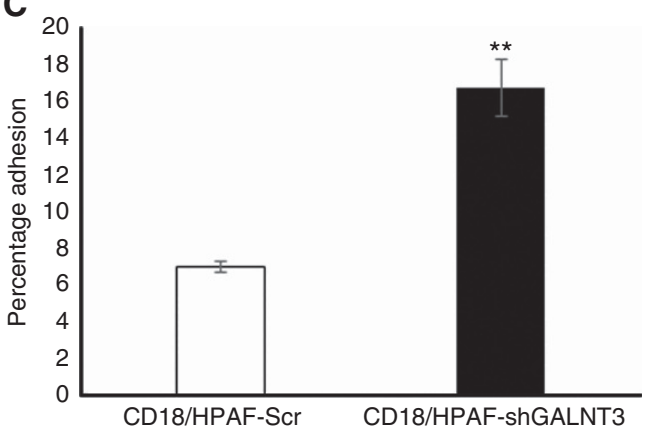

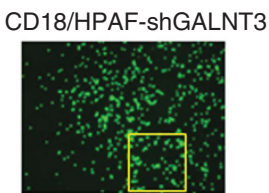

CD18/HPAF-shGALNT3

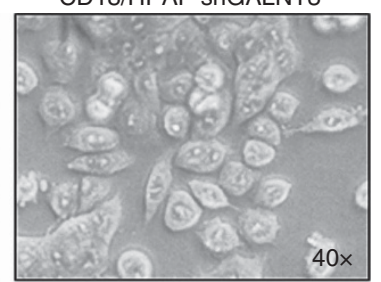

BXPC3-shGALNT3

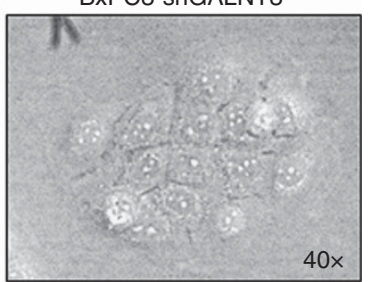

B

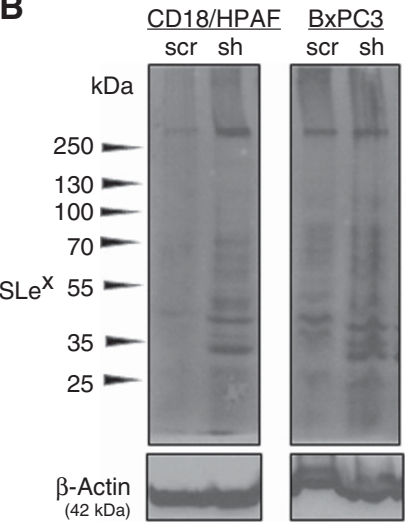

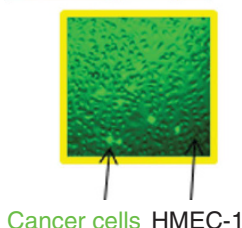

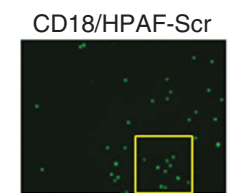

Cancer cells HMEC-1
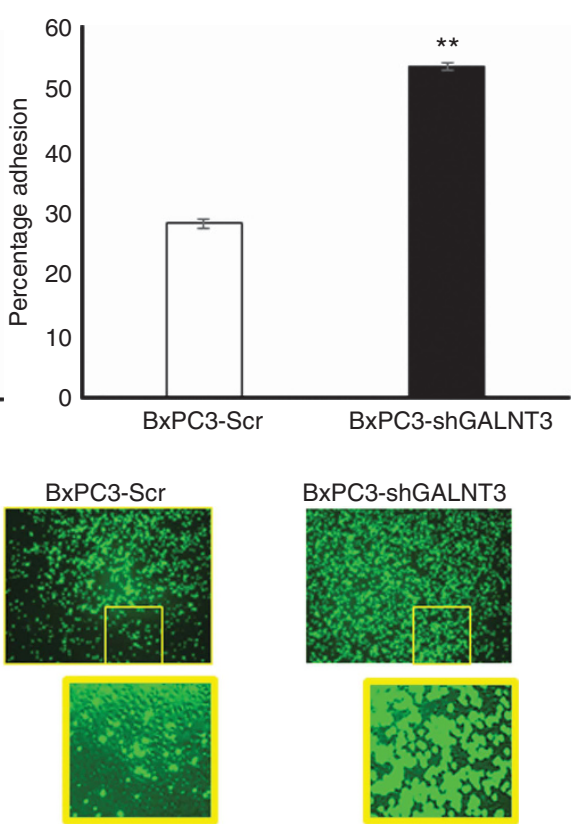

Figure 5. Altered morphology and increased adhesion of GALNT3 knockdown cells to endothelium monolayer. (A) Epithelial-mesenchymal transition-like morphological changes in GALNT3 knockdown CD18/HPAF and BxPC3 PDAC cells. (B) Increased expression of metastatic SLe carbohydrate epitope in GALNT3 knockdown PDAC cells. (C) Bar graphs represent significant increase in percentage adhesion of GALNT3 knockdown cells to endothelial monolayer ( ${ }^{\star *} P<0.001$ ). Representative immunofluorescent images showing increased adhesion of GALNT3 knockdown cells to endothelium monolayer as compared with scrambled control cells (Green-cancer cells).

was to study the impact of GALNT3 loss on pancreatic cancer pathogenesis.

Several studies report associations between GALNT3 expression and clinicopathological features of cancers. For example, in lung adenocarcinoma, decreased expression of GALNT3 is associated with poorly differentiated tumours, poor survival and lymph-node metastasis (Dosaka-Akita et al, 2002; Gu et al, 2004). Similarly, in the case of gastric and colorectal carcinoma, patients with decreased expression of GALNT3 showed poorer survival, whereas patients with strong GALNT3 expression had a good prognosis (Shibao et al, 2002; Onitsuka et al, 2003). Taken together, these studies suggest the loss of GALNT3 expression is associated with the more aggressive forms of cancer. In contrast to these studies, where loss of GALNT3 expression correlates with poor survival, a study in renal cell carcinoma shows that patients with positive GALNT3 cases have poor prognosis (Kitada et al, 2013).
This suggests that the positive/negative correlation of GALNT3 expression with prognosis depends on specific type of tumour.

We next sought to determine the effect of GALNT3 knockdown on the aggressiveness of PDAC cells. Loss of GALNT3 in PDAC cells was accompanied by increased proliferation. Our findings contrast those from a study by Taniuchi et al (2011), wherein data demonstrated that overexpression of GALNT3 leads to increased PDAC growth. In our study, GALNT3 knockdown cells also displayed increased motility, accompanied by altered expression of EMT markers. Interestingly, our results corroborate with previous findings by Kato et al (2010) for hepatocellular carcinoma, wherein cells with increased metastatic potential were marked by decreased expression of GALNT3. In addition, a study by Maupin et al (2010), which focused on identifying glycogene alterations in PDAC EMT, also showed downregulation of GALNT3 in mesenchymal-like PDAC cells. On the basis of this 
A

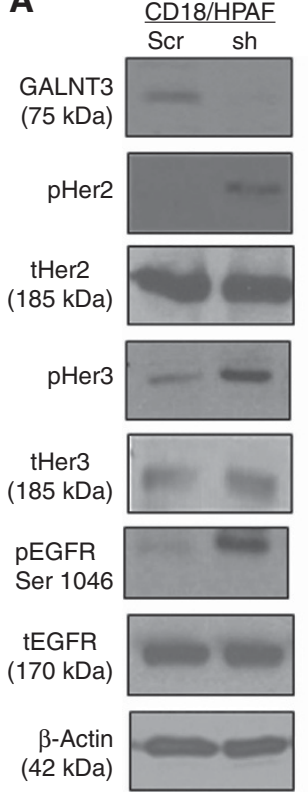

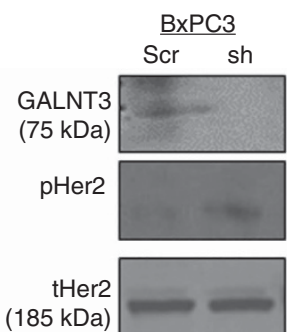

pHer3

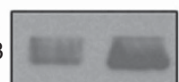

tHer3

(185 kDa)

$\beta$-Actin

(42 kDa)

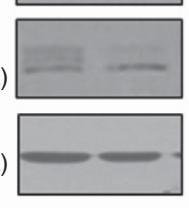

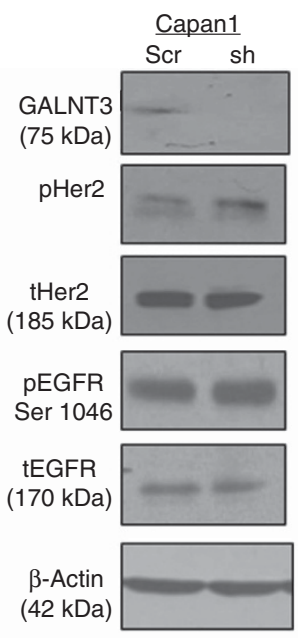

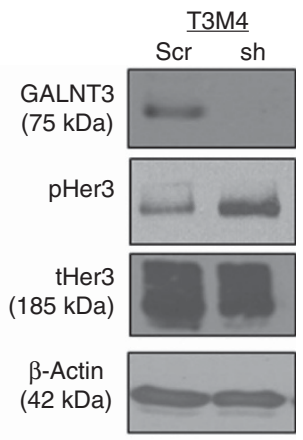

B

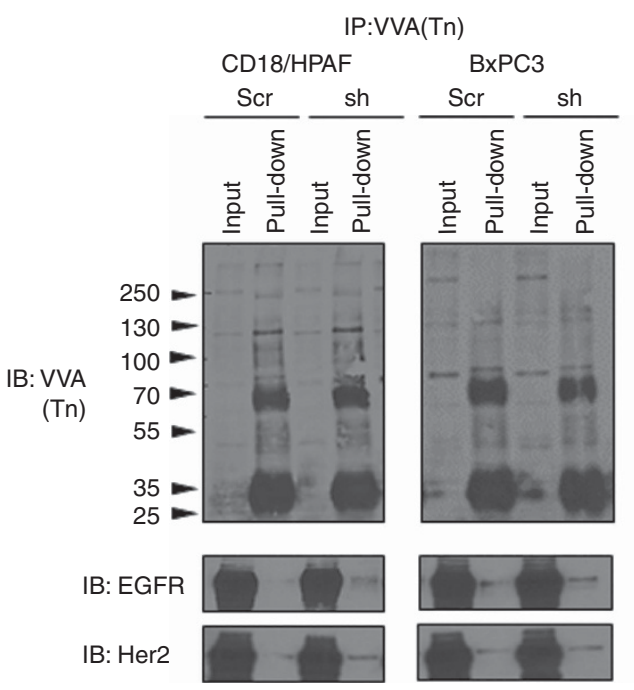

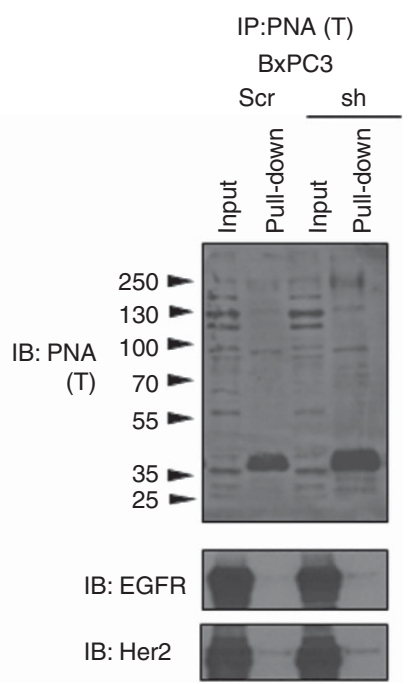

Figure 6. Increased phosphorylation and altered glycosylation of members of the ErbB family of proteins in GALNT3 knockdown cells. (A) GALNT3 knockdown PDAC cells were associated with increase in phosphorylated forms of ErbB family of receptors such as EGFR, Her2 and Her3. (B) Lysates collected from scrambled control and GALNT3 knockdown cells were used for pull-down assays using biotin-conjugated VVA and PNA lectins. Immunoblotting of pulled down precipitates with biotinylated WVA and PNA lectins revealed effective pull down of Tn and T carbohydrate antigens, respectively. Further immunoblotting of pull-down lysates with anti-EGFR and Her2 antibodies demonstrated increase in Tn

carbohydrate antigen association with EGFR and Her2 in GALNT3 knockdown CD18/HPAF and BxPC3 cells compared with scramble control cells. GALNT3 knockdown BxPC3 cells also displayed increased expression of T carbohydrate antigen on EGFR and Her2 as compared with scrambled control cells (IB-Immunoblotting, IP-Immunoprecipitation).

supporting information, our results suggest that GALNT3 loss leads to the aggressiveness of PDAC cells.

Subsequently, we also found increased expression of $\mathrm{SLe}^{\mathrm{x}}$ carbohydrate antigen in GALNT3 knockdown PDAC cells, which was associated with increased percentage adhesion of tumour cells to endothelial cells. Further investigations are necessary to identify the proteins carrying increased expression of SLe ${ }^{\mathrm{x}}$ carbohydrate antigen in GALNT3 knockdown PDAC cells These results indicate that GALNT3 knockdown cells have increased tendency for intravasation/extravasation, which is essential for metastasis. In accordance with results from the functional studies, we also investigated molecular alterations for several members of the ErbB family based on the previous study that showed the critical involvement of ErbB family members in PDAC growth and motility (Roskoski, 2014). Likewise, we observed increased phosphorylation of EGFR, Her2 and Her3 in GALNT3 knockdown PDAC cells. To further delineate the mechanism by which loss of a glycosyltransferase leads to increased phosphorylation of ErbB proteins and increased cancer aggressiveness, lectin pull-down assays were performed to identify the glycan alterations on EGFR and Her2. Recent studies highlight the significant involvement of $O$-glycosylation modifications on EGFR in regulating EGFRmediated oncogenic signalling. For instance, knockdown of GALNT2 in oral cancer has been shown to be associated with altered glycosylation and decreased activation of EGFR (Lin et al, 2014). We identified that loss of GALNT3 in PDAC cells was associated with increased expression of Tn carbohydrate antigens on EGFR and Her2 proteins. Altered glycosylation on these 


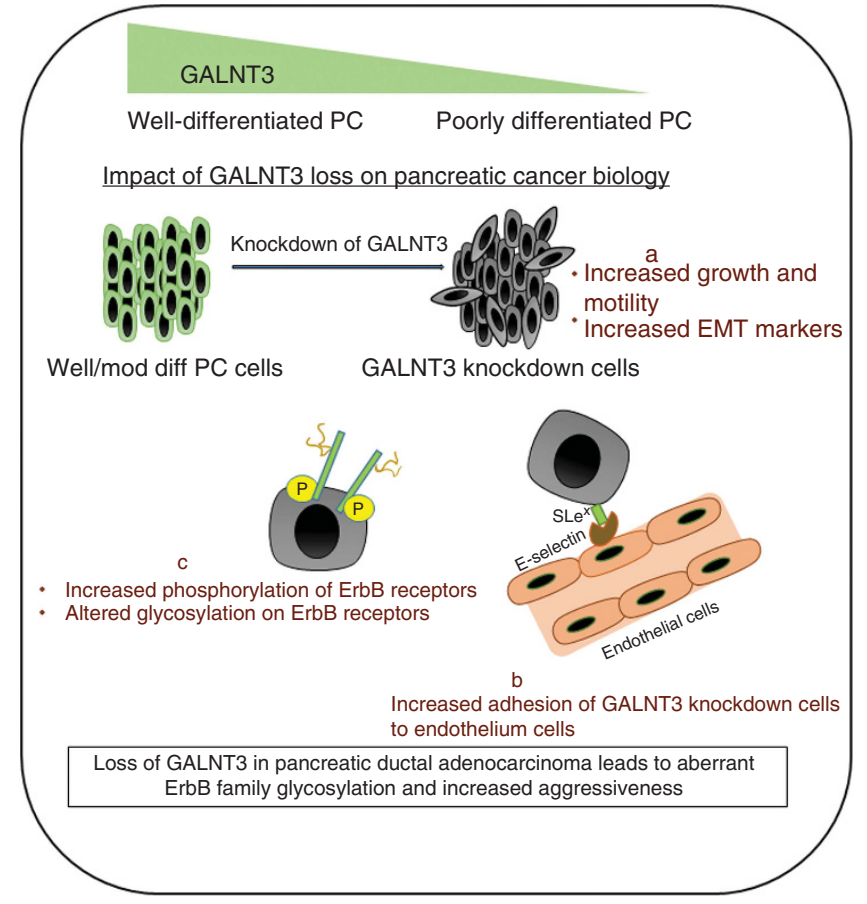

Figure 7. Schematic illustration describing the impact of loss of GALNT3 on PDAC. Reduced expression of GALNT3 was observed in poorly differentiated PDAC tissues as compared with well-differentiated PDAC. Therefore, the functional impact of loss of GALNT3 on PDAC cells was studied by stable knockdown of GALNT3 in PDAC cells. (a) Knockdown of GALNT3 was associated with increased proliferation and motility. (b) Further, GALNT3 knockdown PDAC cells showed increased adhesion to endothelial cells. (c) Increased aggressiveness of GALNT3 knockdown PDAC cells was accompanied by increased phosphorylation of ErbB receptors. Altered glycosylation was also observed on ErbB receptors, which suggests the possible involvement of O-glycans in regulating ErbB signalling. Overall, loss of GALNT3 leads to increased aggressiveness of PDAC cells.

members of the ErbB family of proteins could be a plausible mechanism for their increased activation. Increased expression of carbohydrate antigens on EGFR and Her2 with loss of GALNT3 provides a perspective about compensatory role of other members of GALNT family. The likelihood of such a switch in the expression/activity of GALNTs as disease progresses to an aggressive stage needs to be deciphered.

Importantly, our study shows, for the first time, those $O$-glycan modifications exist on Her2. Prior studies have reported the existence of only $\mathrm{N}$-glycans on Her2 (Watanabe et al, 2013). Using the NetOGlyc 4.0 Server (http://www.cbs.dtu.dk/services/ NetOGlyc/), which predicts mucin-type GalNAc O-glycosylation sites in mammalian protein sequences, we found few potential GalNAc $O$-glycosylation sites in the total Her2. This intriguing finding warrants more experimentation to investigate the exact sites of GalNAc O-glycosylation and the effect of altered glycosylation on the conformation of members of the ErbB family of proteins and their subsequent interaction with other signalling proteins.

Overall, this study shows a novel role of loss of a glycosyltransferase, GALNT3, in PDAC, which is associated with altered glycosylation of ErbB receptors and increased aggressiveness of PDAC (Figure 7). Understanding the mechanism by which altered glycosylation brought by loss of GALNT3 leads to increased activation of EGFR and Her2 has the potential to pave the path for the development of novel and more effective therapeutic regimens.

\section{ACKNOWLEDGEMENTS}

We were supported primarily by the following grants from the National Institutes of Health (RO1 CA183459, RO1 CA195586, TMEN U54CA163120, EDRN UO1 CA111294, SPORE P50CA127297 and K22 CA175260). Seema Chugh is a graduate student supported by Mabel J. Reichenbach Scholarship from University of Nebraska Medical Center. We acknowledge the invaluable technical support from Ms. Kavita Mallya. We also thank Janice A. Tayor and James R. Talaska, at the confocal LaserScanning Microscopy core facility at UNMC, for their support. Finally, we thank Ms. Melody Montgomery for the professional editing of this manuscript.

\section{CONFLICT OF INTEREST}

The authors declare no conflict of interest.

\section{REFERENCES}

Bafna S, Singh AP, Moniaux N, Eudy JD, Meza JL, Batra SK (2008) MUC4, a multifunctional transmembrane glycoprotein, induces oncogenic transformation of NIH3T3 mouse fibroblast cells. Cancer Res 68(22): 9231-9238.

Ben-Porath I, Thomson MW, Carey VJ, Ge R, Bell GW, Regev A, Weinberg RA (2008) An embryonic stem cell-like gene expression signature in poorly differentiated aggressive human tumors. Nat Genet 40(5): 499-507.

Bennett EP, Mandel U, Clausen H, Gerken TA, Fritz TA, Tabak LA (2012) Control of mucin-type O-glycosylation: a classification of the polypeptide GalNAc-transferase gene family. Glycobiology 22(6): 736-756.

Chugh S, Gnanapragassam VS, Jain M, Rachagani S, Ponnusamy MP, Batra SK (2015) Pathobiological implications of mucin glycans in cancer: Sweet poison and novel targets. Biochim Biophys Acta 1856(2): 211-225.

Das S, Batra SK (2015) Pancreatic cancer metastasis: are we being pre-EMTed? Curr Pharm Des 21(10): 1249-1255.

Dosaka-Akita H, Kinoshita I, Yamazaki K, Izumi H, Itoh T, Katoh H, Nishimura M, Matsuo K, Yamada Y, Kohno K (2002) N-acetylgalactosaminyl transferase-3 is a potential new marker for non-small cell lung cancers. Br J Cancer 87(7): 751-755.

Gnanapragassam VS, Jain M, Batra SK (2013) Analysis of tumor-associated mucin glycotopes by Western transfer methods. Methods Mol Biol 980: 331-340.

Gu C, Oyama T, Osaki T, Li J, Takenoyama M, Izumi H, Sugio K, Kohno K, Yasumoto K (2004) Low expression of polypeptide GalNAc

$\mathrm{N}$-acetylgalactosaminyl transferase-3 in lung adenocarcinoma: impact on poor prognosis and early recurrence. Br J Cancer 90(2): 436-442.

Gu L, Vogiatzi P, Puhr M, Dagvadorj A, Lutz J, Ryder A, Addya S, Fortina P, Cooper C, Leiby B, Dasgupta A, Hyslop T, Bubendorf L, Alanen K, Mirtti T, Nevalainen MT (2010) Stat5 promotes metastatic behavior of human prostate cancer cells in vitro and in vivo. Endocr Relat Cancer 17(2): 481-493.

Hakomori S (2002) Glycosylation defining cancer malignancy: new wine in an old bottle. Proc Natl Acad Sci USA 99(16): 10231-10233.

Harada Y, Izumi H, Noguchi H, Kuma A, Kawatsu Y, Kimura T, Kitada S, Uramoto H, Wang KY, Sasaguri Y, Hijioka H, Miyawaki A, Oya R, Nakayama T, Kohno K, Yamada S (2015) Strong expression of polypeptide $\mathrm{N}$-acetylgalactosaminyltransferase 3 independently predicts shortened disease-free survival in patients with early stage oral squamous cell carcinoma. Tumour Biol 36(12): 10003-10004.

Hauselmann I, Borsig L (2014) Altered tumor-cell glycosylation promotes metastasis. Front Oncol 4: 28.

Herreros-Villanueva M, Zhang JS, Koenig A, Abel EV, Smyrk TC, Bamlet WR, de Narvajas AA, Gomez TS, Simeone DM, Bujanda L, Billadeau DD (2013) SOX2 promotes dedifferentiation and imparts stem cell-like features to pancreatic cancer cells. Oncogenesis 2: e61.

Huang MJ, Hu RH, Chou CH, Hsu CL, Liu YW, Huang J, Hung JS, Lai IR, Juan HF, Yu SL, Wu YM, Huang MC (2015) Knockdown of GALNT1 
suppresses malignant phenotype of hepatocellular carcinoma by suppressing EGFR signaling. Oncotarget 6(8): 5650-5665.

Ju T, Otto VI, Cummings RD (2011) The Tn antigen-structural simplicity and biological complexity. Angew Chem Int Ed Engl 50(8): 1770-1791.

Karve TM, Cheema AK (2011) Small changes huge impact: the role of protein posttranslational modifications in cellular homeostasis and disease. J Amino Acids 2011: 207691.

Kato K, Takeuchi H, Kanoh A, Miyahara N, Nemoto-Sasaki Y, Morimoto-Tomita M, Matsubara A, Ohashi Y, Waki M, Usami K, Mandel U, Clausen H, Higashi N, Irimura T (2010) Loss of UDPGalNAc:polypeptide $\mathrm{N}$-acetylgalactosaminyltransferase 3 and reduced $\mathrm{O}$-glycosylation in colon carcinoma cells selected for hepatic metastasis. Glycoconj J 27(2): 267-276.

Kaur S, Momi N, Chakraborty S, Wagner DG, Horn AJ, Lele SM, Theodorescu D, Batra SK (2014) Altered expression of transmembrane mucins, MUC1 and MUC4, in bladder cancer: pathological implications in diagnosis. PLoS One 9(3): e92742.

Kawarada Y, Ishikura H, Kishimoto T, Kato H, Yano T, Kato H, Yoshiki T (2000) The role of sialylated Lewis antigens on hematogenous metastases of human pancreas carcinoma cell lines in vivo. Pathol Res Pract 196(4): 259-263.

Kitada S, Yamada S, Kuma A, Ouchi S, Tasaki T, Nabeshima A, Noguchi H, Wang KY, Shimajiri S, Nakano R, Izumi H, Kohno K, Matsumoto T, Sasaguri Y (2013) Polypeptide N-acetylgalactosaminyl transferase 3 independently predicts high-grade tumours and poor prognosis in patients with renal cell carcinomas. Br J Cancer 109(2): 472-481.

Krueger KE, Srivastava S (2006) Posttranslational protein modifications: current implications for cancer detection, prevention, and therapeutics. Mol Cell Proteomics 5(10): 1799-1810.

Lakshmanan I, Seshacharyulu P, Haridas D, Rachagani S, Gupta S, Joshi S, Guda C, Yan Y, Jain M, Ganti AK, Ponnusamy MP, Batra SK (2015) Novel HER3/MUC4 oncogenic signaling aggravates the tumorigenic phenotypes of pancreatic cancer cells. Oncotarget 6(25): 21085-21099.

Li Z, Yamada S, Inenaga S, Imamura T, Wu Y, Wang KY, Shimajiri S, Nakano R, Izumi H, Kohno K, Sasaguri Y (2011) Polypeptide $\mathrm{N}$-acetylgalactosaminyltransferase 6 expression in pancreatic cancer is an independent prognostic factor indicating better overall survival. $\mathrm{Br} J$ Cancer 104(12): 1882-1889.

Lin MC, Huang MJ, Liu CH, Yang TL, Huang MC (2014) GALNT2 enhances migration and invasion of oral squamous cell carcinoma by regulating EGFR glycosylation and activity. Oral Oncol 50(5): 478-484.

Maupin KA, Sinha A, Eugster E, Miller J, Ross J, Paulino V, Keshamouni VG, Tran N, Berens M, Webb C, Haab BB (2010) Glycogene expression alterations associated with pancreatic cancer epithelial-mesenchymal transition in complementary model systems. PLoS One 5(9): e13002.

Mochizuki Y, Ito K, Izumi H, Kohno K, Amano J (2013) Expression of polypeptide $\mathrm{N}$-acetylgalactosaminyl transferase- 3 and its association with clinicopathological factors in thyroid carcinomas. Thyroid 23(12): $1553-1560$

Nieto J, Grossbard ML, Kozuch P (2008) Metastatic pancreatic cancer 2008: is the glass less empty? Oncologist 13(5): 562-576.

Onitsuka K, Shibao K, Nakayama Y, Minagawa N, Hirata K, Izumi H, Matsuo K, Nagata N, Kitazato K, Kohno K, Itoh H (2003) Prognostic significance of UDP-N-acetyl-alpha-D-galactosamine:polypeptide $\mathrm{N}$-acetylgalactosaminyltransferase-3 (GalNAc-T3) expression in patients with gastric carcinoma. Cancer Sci 94(1): 32-36.

Park JH, Nishidate T, Kijima K, Ohashi T, Takegawa K, Fujikane T, Hirata K, Nakamura Y, Katagiri T (2010) Critical roles of mucin 1 glycosylation by transactivated polypeptide $\mathrm{N}$-acetylgalactosaminyltransferase 6 in mammary carcinogenesis. Cancer Res 70(7): 2759-2769.

Raman J, Fritz TA, Gerken TA, Jamison O, Live D, Liu M, Tabak LA (2008) The catalytic and lectin domains of UDP-GalNAc:polypeptide alpha-NAcetylgalactosaminyltransferase function in concert to direct glycosylation site selection. J Biol Chem 283(34): 22942-22951.
Roskoski Jr. R (2014) The ErbB/HER family of protein-tyrosine kinases and cancer. Pharmacol Res 79: 34-74.

Schultz MJ, Swindall AF, Bellis SL (2012) Regulation of the metastatic cell phenotype by sialylated glycans. Cancer Metastasis Rev 31(3-4): 501-518.

Seales EC, Jurado GA, Singhal A, Bellis SL (2003) Ras oncogene directs expression of a differentially sialylated, functionally altered beta1 integrin. Oncogene 22(46): 7137-7145.

Seshacharyulu P, Ponnusamy MP, Rachagani S, Lakshmanan I, Haridas D, Yan Y, Ganti AK, Batra SK (2015) Targeting EGF-receptor(s)—axis attenuates tumor growth and metastasis through downregulation of MUC4 mucin in human pancreatic cancer. Oncotarget 6(7): 5164-5181.

Shibao K, Izumi H, Nakayama Y, Ohta R, Nagata N, Nomoto M, Matsuo K, Yamada Y, Kitazato K, Itoh H, Kohno K (2002) Expression of UDP-Nacetyl-alpha-D-galactosamine-polypeptide galNAc N-acetylgalactosaminyl transferase- 3 in relation to differentiation and prognosis in patients with colorectal carcinoma. Cancer 94(7): 1939-1946.

Siegel RL, Miller KD, Jemal A (2016) Cancer statistics, 2016. CA Cancer J Clin 66(1): 7-30

St Hill CA (2011) Interactions between endothelial selectins and cancer cells regulate metastasis. Front Biosci (Landmark Ed) 16: 3233-3251.

Stanley P (2011) Golgi glycosylation. Cold Spring Harb Perspect Biol 3(4): a005199.

Steinberg W (1990) The clinical utility of the CA 19-9 tumor-associated antigen. Am J Gastroenterol 85(4): 350-355.

Takahashi S, Oda T, Hasebe T, Sasaki S, Kinoshita T, Konishi M, Ueda T, Nakahashi C, Ochiai T, Ochiai A (2001) Overexpression of sialyl Lewis x antigen is associated with formation of extratumoral venous invasion and predicts postoperative development of massive hepatic metastasis in cases with pancreatic ductal adenocarcinoma. Pathobiology 69(3): 127-135.

Taniuchi K, Cerny RL, Tanouchi A, Kohno K, Kotani N, Honke K, Saibara T, Hollingsworth MA (2011) Overexpression of GalNAc-transferase GalNAc-T3 promotes pancreatic cancer cell growth. Oncogene 30(49): 4843-4854.

Torres MP, Rachagani S, Souchek JJ, Mallya K, Johansson SL, Batra SK (2013) Novel pancreatic cancer cell lines derived from genetically engineered mouse models of spontaneous pancreatic adenocarcinoma: applications in diagnosis and therapy. PLoS One 8(11): e80580.

Tran DT, Ten Hagen KG (2013) Mucin-type O-glycosylation during development. J Biol Chem 288(10): 6921-6929.

Wang ZQ, Bachvarova M, Morin C, Plante M, Gregoire J, Renaud MC, Sebastianelli A, Bachvarov D (2014) Role of the polypeptide $\mathrm{N}$-acetylgalactosaminyltransferase 3 in ovarian cancer progression: possible implications in abnormal mucin O-glycosylation. Oncotarget 5(2): 544-560.

Watanabe M, Terasawa K, Kaneshiro K, Uchimura H, Yamamoto R, Fukuyama Y, Shimizu K, Sato TA, Tanaka K (2013) Improvement of mass spectrometry analysis of glycoproteins by MALDI-MS using 3aminoquinoline/alpha-cyano-4-hydroxycinnamic acid. Anal Bioanal Chem 405(12): 4289-4293.

Wu YM, Liu CH, Hu RH, Huang MJ, Lee JJ, Chen CH, Huang J, Lai HS, Lee PH, Hsu WM, Huang HC, Huang MC (2011) Mucin glycosylating enzyme GALNT2 regulates the malignant character of hepatocellular carcinoma by modifying the EGF receptor. Cancer Res 71(23): 7270-7279.

Yamamoto S, Nakamori S, Tsujie M, Takahashi Y, Nagano H, Dono K, Umeshita K, Sakon M, Tomita Y, Hoshida Y, Aozasa K, Kohno K, Monden M (2004) Expression of uridine diphosphate N-acetyl-alpha-Dgalactosamine: polypeptide $\mathrm{N}$-acetylgalactosaminyl transferase 3 in adenocarcinoma of the pancreas. Pathobiology 71(1): 12-18.

This work is published under the standard license to publish agreement. After 12 months the work will become freely available and the license terms will switch to a Creative Commons AttributionNonCommercial-Share Alike 4.0 Unported License.

Supplementary Information accompanies this paper on British Journal of Cancer website (http://www.nature.com/bjc) 